\title{
Cortical and subcortical glucose metabolism in childhood epileptic encephalopathies
}

\author{
C D Ferrie, P K Marsden, M N Maisey, R O Robinson
}

Department of

Paediatric Neurology,

The General Infirmary

at Leeds, UK

C D Ferrie

Clinical PET Centre, Department of

Radiological Sciences,

UMDS, London, UK

P K Marsden

M N Maisey

Department of

Paediatric Neurology,

Guy's Hospital,

London, UK

R O Robinson

Correspondence to:

Dr C D Ferrie, Department

of Paediatric Neurology,

Clarendon Wing,

The General Infirmary at

Leeds, Belmont Grove,

Leeds, LS2 9NS.

Received 22 October 1996 and in final revised form 9 April 1997

Accepted 21 April 1997

\begin{abstract}
Objectives - Nearly one third of children with cryptogenic epileptic encephalopathies have been reported to have focal cortical defects on ${ }^{18}$ fluorodeoxyglucose (FDG) PET. As diffuse cortical dysfunction and involvement of subcortical structures, particularly the thalami, is postulated to underlie the propensity to seizures in these conditions, the aim was to determine the frequency of bilateral and diffuse cortical metabolic defects and of subcortical metabolic abnormalities in the same patients.

Methods-The interictal uptake of FDG was studied in 32 children with epileptic encephalopathies. Using a semiquantitative technique, the ratio of uptake in cortical regions and subcortical structures to that in the cerebellum was compared with that of age matched historical controls. Uptake more than 2 SD above ("hypermetabolic") or below ("hypometabolic") that of age matched controls was considered abnormal.

Results-Diffusely abnormal cortical uptake (nearly always hypometabolic) occurred in almost two thirds of patients; in all but two of the remaining patients at least one cortical region showed significantly decreased uptake bilaterally. When analysed as age cohorts, the mean cortical:cerebellar FDG uptake was significantly lower than that of controls in all cortical regions $(P<0.005)$. Ninety per cent of patients had evidence of relative thalamic hypometabolism and in each age group there was a significant reduction in relative thalamic FDG uptake compared with that of controls $(P<0.005)$. In nine out of 11 patients with unilateral cortical hypometabolic defects thalamic FDG uptake was lower ipsilateral to the cortical abnormality.

Conclusions-Diffuse cortical dysfunction is common in the epileptic encephalopathies and may reflect the underlying cause of the condition or arise as a consequence of uncontrolled seizures. Altered thalamic glucose metabolism is further evidence of subcortical involvement in these conditions.
\end{abstract}

(F Neurol Neurosurg Psychiatry 1997;63:181-187)

Keywords: positron emission tomography; LennoxGastaut syndrome, infantile spasms, severe myoclonic epilepsy in infancy
The childhood epileptic encephalopathies are drug resistant epilepsies characterised by multiple seizure types and diffusely slow EEG with generalised or multifocal paroxysmal abnormalities. ${ }^{1-4}$ Of all the epilepsies, they have proved the most difficult to classify but syndromes such as the Lennox-Gastaut syndrome (either evolving from West syndrome or arising de novo), severe myoclonic epilepsy in infancy and epilepsy with myoclonic-astatic seizures have been defined. ${ }^{1}$ The extent to which their aetiologies differ and how helpful such classification is in predicting response to treatment or prognosis is unclear. Newer imaging modalities, including functional techniques, may prove useful in this regard.

PET with ${ }^{18}$ fluorodeoxyglucose (FDG) has been used to study patients with infantile spasms and the Lennox-Gastaut syndrome and various patterns of cortical glucose metabolism have been described. ${ }^{5-10}$ In some studies the syndromic definitions used, particularly of the Lennox-Gastaut syndrome, were broad. ${ }^{6}$ We have previously reported FDG PET findings in 32 children with various cryptogenic epileptic encephalopathies which were strictly classified into epilepsy syndromes. ${ }^{11}$ Visual inspection combined with semiquantitative analysis using calculated asymmetry indices for homologous cortical regions were used to detect cortical metabolic defects. Unifocal abnormalities were found in 12 patients and multifocal or bilateral and diffuse abnormalities in a further five. In the remainder, including all patients with typical Lennox-Gastaut syndrome, no abnormality was apparent. The high incidence of apparently normal scans is surprising given that diffuse involvement of the cerebral cortex is considered probable in the epileptic encephalopathies. However, visual inspection and semiquantitative analysis using asymmetry indices are ill suited to detect bilateral and diffuse abnormalities. FDG PET has been suggested as useful in assessing the functional integrity of the remaining cortex in patients being considered for resective epilepsy surgery, ${ }^{6}$ and bilateral temporal hypometabolism is reported as a poor prognostic sign in infantile spasms. ${ }^{12}$ The detection of bilateral and diffuse metabolic abnormalities in children with epileptic encephalopathies is, therefore, clearly desirable.

There is strong evidence that seizure generation in the epileptic encephalopathies reciprocally involves both cortical and subcortical structures. ${ }^{13-15}$ However, abnormalities in children with epileptic encephalopathies have not been shown in the thalami or other subcortical structures by structural imaging and infor- 
mation regarding subcortical metabolism and blood flow is limited apart from the finding of increased FDG uptake particularly in the lentiform nuclei in children with infantile spasms. ${ }^{16}$ Increased ictal subcortical blood flow and glucose metabolism are reported in the idiopathic generalised epilepsies; ${ }^{17}$ interictal studies are generally normal. ${ }^{17}{ }^{18}$ In partial epilepsies, a minority of patients have thalamic glucose hypometabolism ipsilateral to the cortical focus. ${ }^{18}$

Fully quantified techniques for analysing PET data are unpopular in paediatric practice and require each centre to have appropriate age matched controls. Semiquantitative analysis does not involve additional invasive procedures and may not require centre specific control data. ${ }^{19}$ It involves comparing FDG uptake in computer generated regions of interest (ROIs). Focal cortical abnormalities can be detected by comparing radiotracer uptake in ROIs placed in homologous brain regions using calculated asymmetry indices. ${ }^{18}$ Uptake in cortical and subcortical structures can also be expressed as a ratio of that against a common reference such as the cerebellum. Values obtained can then be compared with appropriate control data allowing detection of focal, bilateral, and diffuse cortical defects as well as abnormalities in subcortical structures. Chugani et al have published details on the development of cortical and subcortical FDG uptake from infancy to early adult life obtained in children subsequently proved not to have significant neurological disease. ${ }^{20}$ This provides potential historical control data for FDG PET studies in children.

Here we describe an investigation of cortical and subcortical glucose metabolism in the same group of children in which we previously reported focal cortical metabolic abnormalities detected by visual inspection and semiquantitative analysis using asymmetry indices. Further analysis of the data is made comparing the ratios of cortical:cerebellar and subcortical:cerebellar FDG uptake in patients against age matched historical controls. ${ }^{20}$

\section{Methods}

Thirty two children, age range 1 to 12 years, with cryptogenic epileptic encephalopathies were studied prospectively. In all patients extensive investigation had failed to detect focal cerebral abnormalities. These included detailed EEG and video-EEG studies and MRI with $6 \mathrm{~mm}$ thick axial proton density and T2 weighted spin echo images of the whole head and $3 \mathrm{~mm}$ thick T2 weighted fast spin echo images and $4 \mathrm{~mm}$ thick T1 weighted images orthogonal to the long axis of the temporal lobes. Patients were classified syndromically according to the proposals of the International League Against Epilepsy. ${ }^{1}$ FDG PET (Siemens ECAT 951R PET scanner) with EEG monitoring during radiotracer (3.6 MBq/kg FDG) uptake was performed after a four hour fast. During FDG uptake lights were dimmed and interactions with the child discouraged. Intravenous diazepam was given to patients with frequent paroxysmal EEG abnormalities during the first 10 minutes of FDG uptake and also to agitated patients. PET was performed after 30 minutes, using a head holder to minimise movements. Six five minute consecutive frames were acquired with the data summed. Frames with excessive movement were discarded. After correction for attenuation using a calculated method, ${ }^{21}$ images were smoothed and reconstructed to give $313.4 \mathrm{~mm}$ thick planes with an in plane spatial resolution of 8 $\mathrm{mm} \mathrm{FWHM} \mathrm{(full} \mathrm{width} \mathrm{half} \mathrm{maximum)} \mathrm{and} \mathrm{a}$ total axial field of view of $10.4 \mathrm{~cm}$. Images were reconstructed in axial and coronal planes and in the plane parallel to the long axis of the temporal lobes. The EEG during radiotracer uptake was used to classify scans as exclusively, predominantly, or probably interictal or ictal. Further details are given elsewhere. ${ }^{11}$

Semiquantitative analysis was performed using a template of multiple $4 \mathrm{~mm}$ diameter circular ROIs placed in selected brain regions chosen by matching PET planes to an anatomical brain atlas. Frontal (five ROIs on each side), parietal (three ROIs on each side), occipital (three ROIs on each side), and temporal (four ROIs on each side) cortices were sampled, along with the lenticular nuclei (one ROI on each side), caudate nuclei (one ROI on each side), and thalami (one ROI on each side). Multiple ROIs were averaged. For each cortical region and subcortical structure FDG uptake was expressed relative to that in the cerebellum (three ROIs on each side). The values obtained in each patient were compared with historical age matched control data, ${ }^{20}$ with a ratio of $2 \mathrm{SD}$ or more from the mean of controls being considered abnormal leading to a designation of hypometabolic or hypermetabolic as appropriate. The SD of the mean ratios of cortical and subcortical:cerebellar uptake in controls was calculated by the delta method. ${ }^{22}$ As determined by the age cohorts used in the control data, patients were also separated into age groups; $1-2,3-8$, and 9-12 years and for each age group the mean ratio of uptake in cortical regions and subcortical structures to that in the cerebellum was compared with control data, ${ }^{20}$ using $t$ tests to assess significance. Analysis according to the presence or absence of focal cortical PET abnormalities (as detailed elsewhere $^{11}$ ) was also performed. Given that many comparisons were made, a relatively stringent significance level was used: A P value $<0.005$ was taken to infer that a particular difference was unlikely to have arisen by chance.

In addition asymmetry indices (difference in uptake between homologous structures/ (average uptake in homologous structures) $\times$ $100 \%$ ) were calculated in each patient for homologous subcortical structures. The normal variation in FDG uptake between paired subcortical structures in children is unknown and therefore all asymmetries, however small, were noted. In patients with unifocal cortical hypometabolic defects, asymmetries of subcortical structures were assessed as to whether the side with lesser uptake ("hypometabolic") was ipsilateral or contralateral to the cortical abnormality. As an internal control, in patients without focal cortical abnormalities, asym- 
Table 1 Clinical data on patients (only the major interictal paroxysmal EEG changes are shown)

\begin{tabular}{|c|c|c|c|c|c|}
\hline Patient & Age at seizure onset & Age at PET $(y)$ & Syndrome diagnosis & Seizure types & Interictal EEG \\
\hline 1 & 6 months & 10 & LGS after IS & IS, DA, M, GTCS & Hyps, SSW, MSW \\
\hline 2 & 6 months & 5 & LGS after IS & IS, $M$, T & Hyps, SSW, MSW \\
\hline 3 & 6 months & 10 & LGS after IS & IS, GTCS $\dagger, \mathrm{A}, \mathrm{T}, \mathrm{AtA}, \mathrm{M} \dagger$ & Hyps, SSW, FR \\
\hline 4 & 8 months & 12 & LGS after IS & IS, T, AtA & SSW \\
\hline 5 & 2 months & 11 & ${ }^{\star}$ LGS after IS & IS, AtA, T, GTCS, M & Hyps, SSW, MSW $\ddagger$ \\
\hline 6 & 3 months & 7 & ${ }^{\star}$ LGS after IS & C, IS, T, M, M-A & MSW $\ddagger$ \\
\hline 7 & 3 weeks & 9 & ${ }^{\star}$ LGS after IS & NeoS, IS, T, GTCS & Hyps, SSW \\
\hline 8 & $3 \mathrm{y}$ & 11 & De novo LGS & AtA, A, NCS & SSW \\
\hline 9 & $2 \mathrm{y}$ & 8 & De novo LGS & GTCS, A, T, M†, CS & SSW \\
\hline 10 & $5 \mathrm{y}$ & 8 & De novo LGS & GTCS, A, AtA, T & SSW \\
\hline 11 & $3 \mathrm{y}$ & 9 & De novo LGS & GTCS, A, T, AtA, M† & SSW, FR \\
\hline 12 & $4 \mathrm{y}$ & 12 & De novo LGS & GTCS, A, AtA, T, NCS & SSW, MSW \\
\hline 13 & $3 \mathrm{y}$ & 9 & ${ }^{\star}$ De novo LGS & $\mathrm{T}, \mathrm{A}, \mathrm{AtA}, \mathrm{U}$ & SSW, FR \\
\hline 14 & $3 \mathrm{y}$ & 5 & ${ }^{\star}$ De novo LGS & GTCS, AtA, U & SSW, MSW \\
\hline 15 & $1 \mathrm{y}$ & 5 & ${ }^{\star}$ De novo LGS & C, GTCS, A, M, T, AtA & SSW, FR \\
\hline 16 & $3 \mathrm{y}$ & 10 & ${ }^{\star}$ De novo LGS & A, C, T, AtA, NCS & SSW, MSW \\
\hline 17 & 2 months & 10 & SMEI & C, GTCS, M-A, AtA, T, CS & SW, MSW \\
\hline 18 & 5 months & 6 & SMEI & C, GTCS, M, AtA & SW, MSW \\
\hline 19 & 5 months & 11 & SMEI & CS, GTCS, AtA, T, M & SW \\
\hline 20 & 7 months & 12 & SMEI & CS, GTCS, AtA, CP, M, T† & SW, MSW \\
\hline 21 & 3 months & 3 & SMEI & CS, GTCS, AtA, M & Diffuse slow only \\
\hline 22 & 5 months & 11 & SMEI & GTCS, M, AtA, C & SW, MSW, PCR \\
\hline 23 & 15 months & 4 & ^SMEI & GTCS, AtA, M & SW \\
\hline 24 & 12 months & 10 & ^SMEI & M, GTCS, AtA, T & MSW \\
\hline 25 & 11 months & 12 & ${ }^{\star} \mathrm{EM}-\mathrm{AS}$ & $\mathrm{M}, \mathrm{M}-\mathrm{A}, \mathrm{Ab}, \mathrm{GTCS}, \mathrm{T}+$, NCS & SW \\
\hline 26 & $2 \mathrm{y}$ & 11 & ${ }^{\star} \mathrm{EM}-\mathrm{AS}$ & $\mathrm{M}, \mathrm{GTCS}, \mathrm{M}-\mathrm{A}, \mathrm{Ab}, \mathrm{NCS}$ & SW, MSW, PCR \\
\hline 27 & $7 \mathrm{y}$ & 12 & Unclassified & $\mathrm{U}, \mathrm{T}, \mathrm{AtA}$ & SSW, MSW \\
\hline 28 & $2 \mathrm{y}$ & 5 & Unclassified & GTCS, NCS, CS, M, T, AtA & SSW, MSW \\
\hline 29 & $1 \mathrm{y}$ & 2 & Unclassified & $\mathrm{U}, \mathrm{AtA}, \mathrm{MA}, \mathrm{M}$ & MSW \\
\hline 30 & $1 \mathrm{y}$ & 8 & Unclassified & AtA, DA, T, M, C & SW, MSW, PCR \\
\hline 31 & 9 months & 6 & Unclassified & GTCS, M, AtA, T & MSW \\
\hline 32 & 5 months & 5 & Unclassified & GTCS, T & MSW \\
\hline
\end{tabular}

IS = infantile spasms; LGS = Lennox-Gastaut syndrome; SMEI = severe mtoclonic epilepsy in infancy; EM-AS = epilepsy with myoclonic-astatic seizures; DA = drop attacks (type undetermined); $\mathrm{M}=$ myoclonic; $\mathrm{GTCS}=$ generalised tonic-clonic seizures; $\mathrm{T}=$ tonic; $\mathrm{A}=$ atonic; AtA = atypical absence; $\mathrm{M}$-A = myoatonic; $\mathrm{NeoS}=$ neonatal seizure; NCS = non-convulsive status; $\mathrm{CS}=$ convulsive status; $\mathrm{C}=$ clonic; $\mathrm{Ab}=$ absence; Hyps = hypsarrythmia; SSW = slow spike and wave; $\mathrm{MSW}=$ multiple spike and wave; FR = fast rhythms in sleep; PCR = photoconvulsive response; ${ }^{\star}$ certain features in these patients were considered atypical of the syndrome (Ferrie et $a l^{11}$ ); $†$ implies seizure type was rare; $\ddagger$ no EEG performed during period of active spasms.

metries were assessed as to whether the hypometabolic side was right or left sided. The significance of asymmetries was assessed by the binomial distribution.

\section{Results}

Thirty two children were studied; 18 were boys. The median age at PET was 9 (range $2-12$ ) years. Only one patient was in the 1-2 year age group and so data from this patient were excluded from the analysis of cortical and subcortical glucose metabolism by age group. Seven patients were classified as LennoxGastaut syndrome after infantile spasms, nine as de novo Lennox-Gastaut syndrome, eight as severe myoclonic epilepsy in infancy, two as epilepsy with myoclonic-astatic seizures, and six were unclassified (table 1). Based on clinical findings and EEG during radiotracer uptake, all but one scan was considered as interictal, probably interictal, or predominantly interictal. One scan was considered ictal. Further details of this is given elsewhere. ${ }^{11}$

CORTICAL GLUCOSE METABOLISM

In all but two patients, bilateral hypometabolism involving at least one pair of cerebral lobes was seen. In 21 patients the relative uptake in all cortical regions was more than 2 SD below that in controls (global cortical hypometabolism). In patients with bilateral, but not global cortical hypometabolism, the posterior brain regions were more often affected than the anterior regions; frontal sparing occurred in nine patients, parietal sparing in eight, but temporal and occipital sparing occurred in only three patients each. In three patients bilateral frontal lobe and in one patient bilateral parietal lobe hypermetabolism occurred. Table 2 shows the ratio of cortical:cerebellar FDG uptake in each age cohort of patients and controls. In each cohort relative FDG uptake in all cerebral lobes was significantly lower than that of controls.

Both bilateral and diffuse hypometabolism was seen in all major syndromic groups and diffuse hypometabolism was as likely to occur in those patients with focal cortical metabolic defects as in those in whom such abnormalities had not been detected (see also table 2).

SUBCORTICAL GLUCOSE METABOLISM

There was relative hypometabolism of both thalami in 26 patients $(81 \%)$ and in one thalamus in a further three patients $(9 \%)$. In only two patients $(6 \%)$ was metabolism of both thalami within the normal range. In one patient thalamic hypermetabolism was seen; this was the only patient whose PET was ictal. By contrast relative hypometabolism occurred in both lentiform nuclei in 13 patients (41\%), in one lentiform nucleus in two patients $(6 \%)$, and was within the normal range in 11 patients $(34 \%)$. In six patients $(19 \%)$ metabolism was increased in one or more nuclei. Hypometabolism of both caudate nuclei occurred in 16 patients $(50 \%)$ and in one caudate nucleus in a further patient (3\%). Metabolism was normal in both caudate nuclei in 11 patients (34\%) and was raised in both nuclei in four patients $(13 \%)$.

Table 2 shows the ratios of uptake in the various subcortical structures to that in the cerebellum according to age group in both 
Table 2 Ratio of FDG uptake in patients and historical age matched controls in cortical regions and subcortical structures compared with cerebellar uptake

\begin{tabular}{|c|c|c|c|c|}
\hline Age (y) & Controls & Whole group & $\begin{array}{l}\text { No focal } \\
\text { abnormalities on PET }\end{array}$ & $\begin{array}{l}\text { Focal abnormalities } \\
\text { on PET }\end{array}$ \\
\hline \multicolumn{5}{|l|}{ Frontal cortex: } \\
\hline $1-2(n=4)$ & $1.52(0.27)$ & & & \\
\hline $3-8(n=12)$ & $1.67(0.10)$ & $1.37(0.30)^{\star}$ & $1.28(0.20)$ & $1.50(0.30)$ \\
\hline $9-15(n=6)$ & $1.63(0.07)$ & $1.36(0.34)^{\star}$ & $1.50(0.36)$ & $1.24(0.31)$ \\
\hline \multicolumn{5}{|l|}{$19-30(n=7)$} \\
\hline \multicolumn{5}{|c|}{ Parietal cortex: } \\
\hline $1-2$ & $1.47(0.26)$ & & & \\
\hline $3-8$ & $1.67(0.10)$ & $1.14(0.26)^{\star \star}$ & $1.07(0.16)$ & $1.25(0.32)$ \\
\hline $9-15$ & $1.57(0.07)$ & $1.14(0.32)^{\star}$ & $1.27(0.39)$ & $1.04(0.22)$ \\
\hline $19-30$ & & & & \\
\hline \multicolumn{5}{|c|}{ Occipital cortex: } \\
\hline $1-2$ & $1.67(0.28)$ & & & \\
\hline $3-8$ & $1.75(0.11)$ & $1.18(0.23)^{\star \star}$ & $1.08(0.24)$ & $1.28(0.20)$ \\
\hline $9-15$ & $1.67(0.05)$ & $1.25(0.20)^{\star \star}$ & $1.23(0.23)$ & $1.22(0.19)$ \\
\hline $19-30$ & & & & \\
\hline \multicolumn{5}{|c|}{ Temporal cortex: } \\
\hline $1-2$ & $1.56(0.27)$ & & & \\
\hline $3-8$ & $1.52(0.09)$ & $1.07(0.27)^{\star \star}$ & $0.99(0.19)$ & $1.16(0.35)$ \\
\hline $9-15$ & $1.46(0.06)$ & $1.01(0.26)^{\star \star}$ & $1.12(0.25)$ & $0.92(0.24)$ \\
\hline $19-30$ & & & & \\
\hline \multicolumn{5}{|c|}{ Lentiform nuclei: } \\
\hline $1-2$ & $1.66(0.30)$ & & & \\
\hline $3-8$ & $1.64(0.10)$ & $1.56(0.23)$ & $1.48(0.12)$ & $1.64(0.31)$ \\
\hline $9-15$ & $1.76(0.06)$ & $1.61(0.25)$ & $1.74(0.24)$ & $1.51(0.20)$ \\
\hline $19-30$ & $1.78(0.10)$ & & & \\
\hline \multicolumn{5}{|c|}{ Caudate nuclei: } \\
\hline $1-2$ & $1.48(0.30)$ & & & \\
\hline $3-8$ & $1.51(0.10)$ & $1.43(0.24)$ & $1.30(0.14)$ & $1.57(0.27)$ \\
\hline $9-15$ & $1.63(0.05)$ & $1.42(0.22)$ & $1.55(0.17)$ & $1.32(0.20)$ \\
\hline $19-30$ & $1.71(0.10)$ & & & \\
\hline \multicolumn{5}{|l|}{ Thalami: } \\
\hline $1-2$ & $1.51(0.25)$ & & & \\
\hline $3-8$ & $1.53(0.09)$ & $1.21(0.25)^{\star \star \star}$ & $1.11(0.10)$ & $1.32(0.33)$ \\
\hline $9-15$ & $1.60(0.05)$ & $1.26(0.21)^{\star \star \star}$ & $1.34(0.24)$ & $1.18(0.17)$ \\
\hline $19-30$ & $1.71(0.13)$ & & & \\
\hline
\end{tabular}

$\star \mathrm{P}<0.005 ;{ }^{\star \star} \mathrm{P}<0.0028 ;{ }^{\star \star \star} \mathrm{P}<0.001$

The SDs for the control values were calculated by the delta method ${ }^{22}$. Only one patient (data not shown) was aged 2 to 3 years. Control values for subcortical structures in subjects aged 19-30 years are included as reference is made to them in the discussion.

Table 3 Association of unilateral focal cortical metabolic defects with ipsilateral subcortical "hypometabolism" and between left and right sided subcortical "hypometabolism" in patients without unilateral focal cortical patients

\begin{tabular}{lccc}
\hline & Lentiform nuclei & Caudate nuclei & Thalami \\
\hline Unilateral cortical hypometabolism: & & & \\
$\quad$ Ipsilateral hypometabolism & 6 & 4 & 9 \\
Contralateral hypometabolism & 5 & 0 & 2 \\
$\quad$ No asymmetry & 0 & 10 & 0 \\
No unilateral cortical metabolic defects: & 11 & 9 & 9 \\
Left sided hypometabolism & 7 & 1 & 0 \\
Right sided hypometabolism & 2 & & 0 \\
No asymmetry & & & \\
\end{tabular}

The difference between ipsilateral and cantralateral thalamic hypometabolism was not significant (see text).

patients and controls. In the group of children as a whole thalamic uptake was significantly less than in controls $(\mathrm{P}<0.001)$ in both age groups; no other significant abnormalities were found. Reduced thalamic uptake could not be related to the presence or absence of focal PET abnormalities or to epilepsy syndrome diagnosis. However, whereas relative metabolism in subcortical structures rose with age in both control subjects and patients without focal PET abnormalities, in patients with focal PET abnormalities the reverse was seen.

Table 3 shows the numbers of children with hypometabolism of subcortical structures ipsilateral and contralateral to focal cortical metabolic defects along with the lateralisation of subcortical hypometabolism in children without focal cortical abnormalities. There was a non-significant $(P=0.065)$ tendency for tha- lamic hypometabolism ipsilateral to cortical hypometabolism.

\section{Discussion}

This study found that almost two thirds of children with epileptic encephalopathies had evidence of diffuse cortical hypometabolism with most of the remainder having hypometabolism affecting at least one cortical lobe bilaterally. This had rarely been suspected on visual inspection. In addition, it is the first to provide evidence from FDG PET of thalamic involvement in the childhood epileptic encephalopathies.

\section{METHODOLOGICAL ISSUES}

The methodology on which these findings are based is indirect as ethical considerations precluded obtaining our own control data. The use of published control data necessitated estimation of the SD of cortical and subcortical:cerebellar FDG uptake in controls. The method used to do this requires that the two elements of the ratio (cortical and cerebellar uptake) are independent. ${ }^{22}$ Moreover, the use of $t$ tests assumes that the two population SDs are equal. We cannot confirm that these requirements were met. However, the consistency of the diffuse cortical and thalamic hypometabolism along with the very small significance levels obtained when comparing age matched groups of patients with controls supports the validity of our findings.

Comparing radiotracer uptake measured on different PET scanners is problematic. Partial volume effects cause measured values to be lower than is actually the case..$^{23-33}$ This is most pronounced in small structures and with low resolution PET scanners. When metabolic rates for glucose obtained from two different PET scanners were compared, partial volume effects were less when studying large structures such as whole cerebral lobes. Additionally, values obtained by the two scanners were generally comparable when FDG uptake was expressed as a ratio against a common denominator (mean grey matter uptake). ${ }^{27}$ Another study on the effects of image resolution on the metabolic rates for prefrontal glucose metabolism showed that, unlike the metabolic values themselves, levels of significance describing group differences of metabolic values were relatively insensitive to spatial resolution. ${ }^{29}$ The intrinsic resolution of the scanner used to study our patients $(6 \mathrm{~mm})$ was greater than that used to study controls (8.4 $\mathrm{mm}$ ), and our slices were significantly thinner $(3.4 \mathrm{~mm} v 12.4 \mathrm{~mm}) .{ }^{20}$ Partial volume effects in the cerebral cortices and subcortical structures are generally greater than in the cerebellum. ${ }^{34}$ Large, irregular ROIs drawn to fit the structures under investigation such as were used to study controls are more susceptible to partial volume effects than small geometric ROIs such as we used..$^{29}$ Overall, partial volume effects would be expected to lower values for the ratio of both cortical and subcortical:cerebellar FDG uptake in controls more than in our patients; the opposite to that which was found. 
The physiological state of subjects during radiotracer uptake can profoundly influence cortical uptake; active patients will generally have higher metabolic rates than those scanned under resting conditions. However, it is unlikely that this explains the widespread cortical hypometabolism found in our patients as they were studied under very similar conditions to those described for controls. They were, however, generally on antiepileptic drugs and some were given diazepam during the radiotracer uptake period. Antiepileptic drugs including the acute administration of diazepam reduce cerebral FDG uptake. ${ }^{35-38}$ However, the effect is diffuse and again is unlikely to explain our findings.

Reduced ratios of cortical and subcortical: cerebellar uptake could be caused by cerebellar hypermetabolism. There is histological evidence of cerebellar involvement in some patients with Lennox-Gastaut syndrome ${ }^{39}$ and cerebellar metabolism is increased during some seizures. ${ }^{40}$ However, it is unlikely that the types of histological abnormality described would give rise to hypermetabolism and the interictal state, experienced by all but one of our patients during FDG uptake, is not associated with cerebellar hypermetabolism.

CORTICAL METABOLISM

The most likely explanation for our findings is that the childhood epileptic encephalopathies are associated with widespread cortical hypometabolism. This supports a previous quantitative FDG PET study on the LennoxGastaut syndrome. ${ }^{7}$ Moreover, it is consistent with the widespread cortical dysfunction postulated to underlie these conditions. Diffuse hypometabolism may reflect either a widespread structural or functional abnormality of the cortex, causally linked to the epileptic encephalopathy or arising as a consequence of uncontrolled seizures. Widespread structural abnormalities, unless subtle, are likely to be detected by MRI. However, microdysgenesis, beyond the resolution of MRI, has been described in children with seizures. ${ }^{39-41}$ Alternatively, uncontrolled seizures could interfere with dendritic arborisation and remodelling which occurs throughout childhood, causing a widespread secondary cortical abnormality, augmenting and maintaining the propensity to seizures. Such abnormalities have been described in a frontal brain biopsy of a child with cryptogenic Lennox-Gastaut syndrome. ${ }^{42}{ }^{43}$

Epileptogenicity can be considered an imbalance between cortical excitation and inhibition. Decreased cortical inhibition could be caused by primary dysfunction of cortical GABAergic inhibitory interneurons. This could cause diffuse cortical hypometabolism depending on the balance of energy requirements between the interneurons and the cortical neurons on to which they project. Alternatively, increased ascending inhibition-for example, in dopaminergic or noradrenergic pathways $^{44}$ - could lead to diffuse cortical hypometabolism. This, however, would probably be associated with hypermetabolism of the subcortical structures giving origin to such projections; our evidence suggests that the contrary occurs. Decreased cortical excitation due to diminished activity of ascending excitatory (for example, acetylcholinergic) pathways would also cause cortical hypometabolism and is compatible with thalamic hypometabolism.

FDG PET has been proposed as being useful in assessing the integrity of the cortex remote from presumptive surgical foci. ${ }^{6}$ If diffuse cortical hypometabolism indicates widespread structural or functional cortical abnormality causing or maintaining the propensity to seizures, surgery is unlikely to improve seizure control. However, if it arises secondary to uncontrolled seizures, then it may be partially reversible if surgical control of seizures is achieved. Some cortical metabolic abnormalities are reported to recover in children with epileptic encephalopathies in whom seizures subside or are controlled medically. ${ }^{45} 46$

SUBCORTICAL METABOLISM

Our finding supports thalamic involvement in the childhood epileptic encephalopathies. The thalamus can generate oscillatory rhythms which have clear relevance to both normal EEG rhythms and paroxysmal epileptiform activity; generalised seizures may involve reverberating circuits between thalami and cortex. ${ }^{47}$ In addition, the reciprocal projections between the thalami and the cortex modulate seizure activity. The thalamus is part of the ascending noradrenergic, dopaminergic, and acetylcholinergic neurotransmitter systems. ${ }^{44}$ Thalamic hypometabolism may reflect dysfunction in such systems leading to a propensity to seizures as discussed previously.

Ipsilateral thalamic hypometabolism has been described in partial epilepsies. ${ }^{18}{ }^{48}$ In our patients overall, relative thalamic hypometabolism was not preferentially associated with the presence or absence of focal cortical FDG PET abnormalities. However, in the epileptic encephalopathies secondary to discrete cortical abnormalities preferential ipsilateral thalamic involvement seems likely (although it did not reach significance). Indeed preferential involvement of the ipsilateral basal ganglia as well is suggested by the reversal of the expected increase with age in relative FDG uptake in lentiform and caudate nuclei as well as in the thalami in patients with focal cortical metabolic defects.

The findings of this study differ from those of a previous study of children with active or recently active infantile spasms, ${ }^{16}$ which showed increased metabolic activity in the brain stem and lentiform nuclei; decreased thalamic activity was not seen. However, as over half of the children were studied using a PET scanner with significantly improved resolution than that used for controls, the higher metabolic rates for the lentiform nuclei could reflect partial volume effects. ${ }^{25-27} 3031$ Alternatively, the different findings of the studies may reflect different stages in the evolution of the "age dependent" epileptic encephalopathies. Theodore et al reported a quantitative study of FDG PET in older children and adults with 
Lennox-Gastaut syndrome. ${ }^{7}$ The metabolic rates for glucose obtained for the caudate nuclei and thalami (the lentiform nuclei were not examined) relative to those for the cerebellum were $1.6(0.3)$ and $1.3(0.3)$ respectively. When compared with the nearest aged matched historical controls (table 2), there is again evidence of thalamic but not caudate hypometabolism. However, the difference failed to reach significance $(0.1>\mathrm{P}>0.05)$, probably reflecting the small sample size.

\section{SYNDROMIC CONSIDERATIONS}

Previously we have shown that typical de novo Lennox-Gastaut syndrome differs from the other syndromes studied here in that it was not associated with focal areas of cortical hypometabolism. ${ }^{11}$ The present study did not show qualitative syndromic differences in either the occurrence of diffuse cortical hypometabolism or in thalamic hypometabolism. The limited numbers in each syndromic group precluded formal statistical analysis. However, overall severe myoclonic epilepsy in infancy and Lennox-Gastaut syndrome after infantile spasms generally had more pronounced reductions in cortical FDG uptake than had those with de novo Lennox-Gastaut syndrome or were unclassified. It is unclear whether this is relevant to the aetiology of these conditions. If, however, cortical hypometabolism arises as a result of uncontrolled seizure activity, more pronounced hypometabolism in the former conditions may simply reflect their earlier age of onset.

\section{Conclusions}

Semiquantitative analysis of FDG PET in which the ratio of cortical:cerebellar uptake was compared with that of historical controls suggested that most children with epileptic encephalopathies have widespread cortical dysfunction. It is not known if this reflects the primary abnormality causing these conditions or if it is a secondary phenomenon caused by uncontrolled seizures. Our study also provides in vivo support for experimental evidence implicating the thalamus in the epileptic encephalopathies. PET using neurotransmitter ligands may shed further light on the nature of thalamic involvement in these conditions.

This study was supported by grants from the Special Trustees of Guy's Hospital and Marion Merrell Dow and Co.

1 Commission on Classification and Terminology of the International League Against Epilepsy. Proposal for revised classification of epilepsies and epileptic syndromes. Epilepsia 1989;30:389-99.

2 Aicardi J. Epileptic encephalopathies of early childood. Current Opinion in Neurology and Neurosurgery 1992;5:344-8.

3 Donat JF. The age-dependent epileptic encephalopathies. $\mathcal{F}$ Child Neurol 1992;7:7-21.

4 Aicardi J. Epilepsy in children. New York: Raven Press, 1994.

5 Gur RC, Sussman NM, Alavi A, et al. Positron emission tomography in two cases of childhood epileptic encephalopathy (Lennox-Gastaut syndrome). Neurology 1982;32 1191-4.

6 Chugani HT, Mazziotta JC, Engel J, Phelps ME. The Lennox-Gastaut syndrome: metabolic subtypes determined by 2-deoxy-2-fluro-D-glucose positron emission mined by 2-deoxy-2-fluro-D-glucose

7 Theodore WH, Rose D, Patronas N, et al. Cerebral glucose metabolism in the Lennox-Gastaut syndrome. Ann Neurol 1987;21:14-21.
8 Yani K, Linuma K, Matsuzawa T, et al. Cerebral glucose utilization in pediatric neurological disorders determined by positron emission tomography. Eur F Nucl Med 1987;13 $292-6$.

9 Miyauchi T, Nomura Y, Ohno S, Kishimoto H, Matsushita M. Positron emission tomography in three cases of Lennox-Gastaut syndrome. Fapanese fournal of Psychiatry and Neurology 1988;42:795-804.

10 Chugani HT, Shields WD, Shewmon DA, Olson DM, Phelps ME, Peacock WJ. Infantile spasms. I. PET identifies focal cortical dysgenesis in cryptogenic cases for surgical treatment. Ann Neurol 1990;27:406-13.

11 Ferrie CD, Maisey M, Cox T, et al. Focal abnormalities detected by ${ }^{18}$ FDG PET in epileptic encephalopathies. Arch Dis Child 1996;75:102-7.

12 Chugani HT, Da Silva E, Chugani DC. Infantile spasms: III. Prognostic implications of bitemporal hypometabolism on positron emission tomogrpahy. Ann Neurol 1996;39: on positro

13 Niedermeyer E. Abnormal EEG patterns: epileptic and paroxysmal. In: Niedermeyer E, Da Silva L, eds. Electroencephalography: basic principles, clinical applications, and related fields. Baltimore: Williams and Wilkins, 1993:217-40.

14 Niedermeyer E. Epileptic seizure disorders. In: Niedermeyer E, Da Silva L, eds. Electroencephalography: basic principles, clinical applications, and related fields. Baltimore: Williams and Wilkins, 1993:461-564.

15 Blume WT. The EEG features of the Lennox-Gastaut syndrome. In: Niedermeyer E, Degan R, eds. The Lennox Gastaut syndrome. New York: Alan R Liss, 1988:159-76.

16 Chugani HT, Shewmon DA, Sankar R, Chen BC, Phelps ME. Infantile spasms: II. Lenticular nuclei and brain stem activation on positron emission tomogrpahy. Ann Neurol 1992;31:212-9.

17 Prevett MC, Duncan JS. Functional imaging studies in humans. In: Duncan JS, Panayiotopoulos CP, et al, eds. hymans. In: Duncan absences and related epileptic syndromes. London: Typical absences and related epileptic syndror

18 Theodore WH. Epilepsy. In: Mazziotta JC, Gilman S, eds. Clinical brain imaging: principles and applications. Philadelphia: FA Davis, 1992:136-65.

19 Sadzot B, Debets R, Maquet P, Comar C, Franck G. PET studies of patients with partial epilepsy: visual Neurol Scand 1994;(suppl 152):175-8.

20 Chugani HT, Phelps ME, Mazziotta JC. Positron emission tomography study of human brain functional development. Ann Neurol 1987;22:487-97.

21 Bergstrom M, Litton J, Erihsson L, Bohm L, Blomquist G. Determination of object contour from projections for attenuation correction in cranial positron emission tomography. F Comput Assist Tomogr 1982;6:365-72.

22 Armitage P. Statistical methods in medical practice. Oxford: Blackwell, 1971

23 Kessler RM, Ellis JR, Eden M. Analysis of emission omographic scan data: limitations imposed by resolution and background. $\mathcal{F}$ Comput Assist Tomogr 1984;8:514-22.

24 Brooks RA, Hatazawa J, Di Chiro G, Larson SM, Fishbein DS. Human cerebral glucose metabolism determined by positron emission tomography: a revisit. $\mathcal{F}$ Cereb Blood Flow Metab 1987;7:427-32.

25 Strother SC, Allard C, Moeller JR, et al. Methodological factors affecting patterns of regional cerebral glucose metabolism as determined by ${ }^{18} \mathrm{~F}$-flurodeoxyglucose/ positron emission tomography. F Cereb Blood Flow Metab positron emission to

26 Grady CL, Berg C, Carson RE, Daube-Witherspoon ME, Friderich RP, Rapoport SI. Quantitative comparison of cerebral glucose metabolic rates from two positron emission tomographs. $\mathcal{F}$ Nucl Med 1989;30:1386-92.

27 Grady CL. Quantitative comparison of measurements of cerebral glucose metabolic rate made with two positron cameras. F Cereb Blood Flow Metab 1991;11(suppl 1):5763.

28 Strother SC, Liow J, Moeller JR, Sidtis JJ, Dhawan VJ, Rottenberg DA. Absolute quantification in neurological PET: do we need it? F Cereb Blood Flow Metab 1991;11(suppl 1):3-16.

29 Kuwert T, Sures T, Herzog H, et al. On the influence of spatial resoloution and of the size and form of regions of interest on the measurement of regional cerebral metabolic rates by positron emission tomography. F Neural Transm Suppl 1992;37:53-66.

30 Kuwert T, Ganslandt T, Jansen P, et al. Influence of size of regions of interest on PET evaluation of caudate glucose metabolism. F Comput Assist Tomogr 1992;16:789-94.

31 Camargo EE, Szabo Z, Links JM, Sostre S, Dannals RF, Wagner HN. The influence of biological and technical factors on the variability of global and regional brain metabolism of 2-[18F] fluoro-2-deoxy-D-glucose. F Cereb Blood Flow Metab 1992;12:281-90.

32 Kumar A, Braun A, Schapiro M, Grady C, Carson R, Herscovitch P. Cerebral glucose metabolic rates after 30 and 45 minute acquisitions: a comparative study. 7 Nucl Med 1992;33:2103-5.

33 Grady CL. Quantitative comparison of measurements of cerebral glucose metabolic rate made with two positron cameras. F Cereb Blood Flow Metab 1991;11(suppl 1):5763. 
34 Hoffmann EJ, Huang S-C, Phelps ME. Quantitation in positron emission tomography I: effect of object size. $\mathcal{F}$ Comput Assist Tomogr 1979;3:299-308.

35 Theodore WH, DiChiro G, Margolin R, Fishbein D, Porter RJ, Brooks RA. Barbiturates reduce human cerebral glucose metabolism. Neurology 1986;36:60-4.

36 Leiderman DB, Balish M, Bromfield EB, Theodore WH. Effect of valproate on human cerebral glucose metabolism. Epilepsia 1991;32:417-22.

37 Theodore WH. Antiepileptic drugs and cerebral glucose metabolism. Epilepsia 1988;29(suppl 2):48-55.

38 Foster NL, VanDerSpek AFL, Aldrich MS, et al. The effect of diazepam sedation on cerebral glucose metabolism in Alzheimer's disease as measured using positron emission tomography. $\mathcal{F}$ Cereb Blood Flow Metab 1987;7:415-20.

39 Roger J, Gambarelli-Dubois D. Neuropathological studies of the Lennox-Gastaut syndrome. In: Niedermeyer E, Degan R, eds. The Lennox-Gastaut syndrome. New York: Alan R Liss, 1987:73-94.

40 Theodore WH, Fishbein D, Deitz M, Baldwin P. Complex partial seizures: cerebellar metabolism. Epilepsia $1987 ; 28$ 319-23.

41 Farrell K. Secondary generalised epilepsy and the LennoxGastaut syndrome. In: Wyllie $\mathrm{E}$, ed. The treatment of epilepsy: principles and practice. Philadelphia: Lea and Febiger, 1993:604-13.
42 Renier WO, Gabreels FJM, Jasper HHJ. Morphological and biochemical analysis of a brain biopsy in a case of idiopathic Lennox-Gastaut syndrome. Epilepsia 1988;29: 644-9.

43 Renier WO. Neuromorphological and biochemical analysis of a brain biopsy in a second case of idiopathic Lennox-Gastaut syndrome. In: Niedermeyer E, Degan R, eds. The Lennox-Gastaut syndrome. New York: Alan R Liss, 1988:427-32.

44 Johnston MV. Neurotransmitters and epilepsy. In: Wyllie E, ed. The treatment of epilepsy: principles and practice. Philadelphia: Lea and Febiger, 1993:111-25.

45 Maeda N, Watanabe K, Negoro T, et al. Evolutional changes of cortical hypometabolism in West's syndrome. Lancet 1994;343:1620-3.

46 Chugani HT, Shewmon DA, Shields WD, et al. Surgery for infantile spasms: neuroimaging perspectives. Epilepsia 1993;34:764-71.

47 Steriade M. Cellular substrates of brain rhythms. In: Niedermeyer E, Da Silva L, eds. Electroencephalography: basic principles, clinical applications, and related fields. Baltimore: principles, clinical applications, and relat

48 Henry TR, Mazziotta JC, Engel J, et al. Quantifying interictal metabolic activity in human temporal lobe epilepsy. $\mathcal{f}$ Cereb Blood Flow Metab 1990;10:748-57. 